\title{
High-Aspect-Ratio SU-8-Based Optofluidic Device for Ammonia Detection in Cell Culture Media
}

\author{
Esma Dervisevic ${ }^{\mathrm{a}}$, Nicolas H. Voelcker ${ }^{\mathrm{b}, \mathrm{c}, \mathrm{d}}$, Gail Risbridger,e,g, \\ Kellie L. Tuck ${ }^{\mathrm{h}}$, Victor J. Cadarso ${ }^{\mathrm{a}, \mathrm{c} *}$.
}

${ }^{a}$ Department of Mechanical and Aerospace Engineering, Monash University, Clayton, Victoria 3800, Australia;

${ }^{\mathrm{b}}$ Monash Institute of Pharmaceutical Sciences (MIPS), Monash University, 381 Royal Parade, Parkville, Victoria 3052, Australia;

${ }^{\mathrm{c}}$ The Melbourne Centre for Nanofabrication, Clayton, Victoria 3800, Australia;

${ }^{\mathrm{d}}$ Commonwealth Scientific and Industrial Research Organization (CSIRO), Clayton, Victoria 3168, Australia;

e Prostate Cancer Translational Research Laboratory, Peter MacCallum Cancer Centre, Melbourne, Victoria 3000, Australia;

${ }^{\mathrm{f}}$ Cancer Program, Biomedicine Discovery Institute and Department of Anatomy and Developmental Biology, Monash University, Clayton, Victoria 3800, Australia;

g Sir Peter MacCallum Department of Oncology, University of Melbourne, Parkville, Victoria 3010, Australia;

${ }^{\mathrm{h}}$ School of Chemistry, Monash University, Clayton, Victoria 3800, Australia.

\section{Corresponding Author}

*Email: victor.cadarso@monash.edu (Victor J. Cadarso), Ph: 0399059211.

\section{Present Address}

Department of Mechanical and Aerospace Engineering, Monash University, Room 227, New Horizons Building, 20 Research Way, Clayton VIC 3800, Australia. 


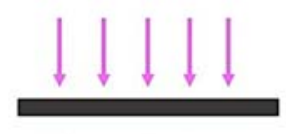

Plasma cleaning

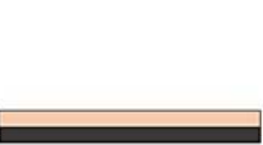

Soft bake 2

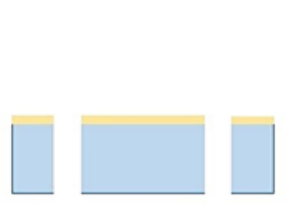

SU-8 spin coating

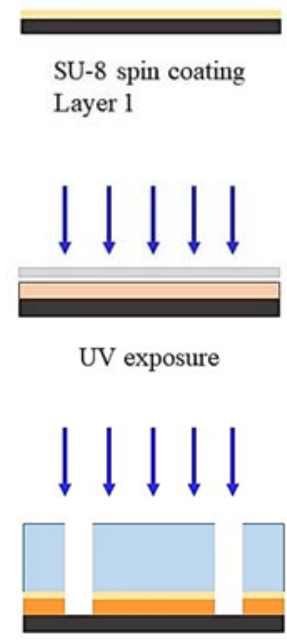

UV exposure

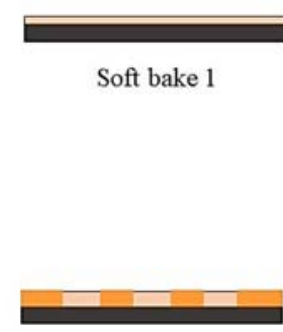

Post exposure bake

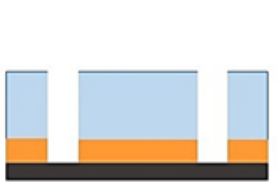

Hard bake
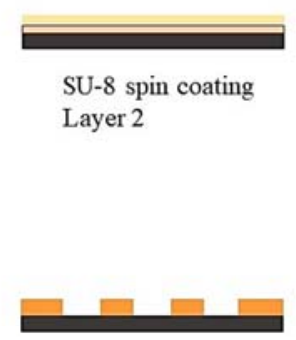

Development

$\square \quad$ Si wafer

SU-8

Soft-baked SU-8

Glass mask

Post exposure-baked SU-8

PDMS

Figure S1: Schematic representation of the fabrication process of SU-8 microfluidic device.

The steps followed during the fabrication of SU-8-based ammonia $\left(\mathrm{NH}_{3}\right)$ sensing device are illustrated in Fig. S1. SU-8 microstructures were fabricated on a Si wafer after which SU-8-coated polydimethylsiloxane (PDMS) was used to form a fully enclosed optical waveguide.

Table S1 shows the results of the analytical recovery study performed with 7 and 40 $\mu \mathrm{M} \mathrm{NH}$. These two concentrations were not used for the calibration plot experiments. The percentage error was calculated using the following formula: $\left|100-\left(\frac{[\text { calculated }]}{[\text { standard }]}\right) * 100\right|$. The maximum error of $9 \%$ demonstrate good analytical performance of the optofluidic ammoniasensing device.

Table S1: Results obtained from the analytical recovery study. SD refers to standard deviation.

\begin{tabular}{|c|c|c|c|}
\hline $\begin{array}{c}\text { Standard - } \\
\boldsymbol{\mu M}\end{array}$ & $\begin{array}{c}\text { Calculated - } \\
\boldsymbol{\mu M}\end{array}$ & $\begin{array}{c}\text { SD }-\boldsymbol{\mu M} \\
(\mathbf{n = 3})\end{array}$ & $\begin{array}{c}\text { Error } \\
\mathbf{( \% )}\end{array}$ \\
\hline 7 & 6.7 & 2.4 & 4.3 \\
\hline 40 & 36.4 & 2.3 & 9.0 \\
\hline
\end{tabular}

